\title{
Biomechanical study of upper-limb exoskeleton for resistance training with three-dimensional motion analysis system
}

\author{
Tzong-Ming Wu, PhD; Dar-Zen Chen, PhD* \\ Department of Mechanical Engineering, National Taiwan University, Taipei, Taiwan (Republic of China)
}

\begin{abstract}
The world's population is aging rapidly, particularly in developed countries. The trend toward prolonged life expectancy will increase the elderly population and thereby lead to an increase in occurrences of age-related health problems such as chronic disease. Healthcare services and homebased rehabilitation are in high demand, and the demand for professional physical therapy is imposing an increasing burden on the healthcare system. Rehabilitation training devices must keep pace with standards of care, be cost effective, and meet the home-based training requirements of today's rehabilitation trends. This article presents an experimental study of a novel spring-loaded upper-limb exoskeleton meant to enable a patient or nondisabled individual to move a limb at multiple joints in different planes for resistance training in a free and unconstrained environment. To assess the functionality of the design, we have measured its kinematic data while performing designated movements and adopted a motion-capture system to verify the function of our mechanism. The collected data and analysis of the kinematic and dynamic joint torques may not only verify our mechanism but also provide a profound understanding of the design requirements for an appropriate springloaded exoskeleton for upper-limb resistance training.
\end{abstract}

Key words: dumbbell, exoskeleton, free-weight exercise, joint torque, motion analysis, rehabilitation, resistance training, SLERT, upper limb, zero-free-length spring.

\section{INTRODUCTION}

According to the World Health Organization, by the year 2050, the number of people over $65 \mathrm{yr}$ old will increase by 73 percent in developed countries and by
20.7 percent worldwide [1]. This age group is particularly prone to stroke. An increasing number of elderly people and changes to their lifestyles have led to an increase in age-related health problems other than stroke, such as chronic diseases. As a result, healthcare services and home-based rehabilitation are in high demand, and the demand for professional physical therapy is imposing an increasing burden on the healthcare system. Healthcare and rehabilitation robotic training devices have the potential to be valuable tools for rehabilitation therapy but must keep pace with both standards of care and the cost-effectiveness of today's rehabilitation trends [2].

The number of technological options for the upperlimb rehabilitation training of patients with stroke has dramatically increased since the upper-limb rehabilitation training robot MIT-Manus was created at the Massachusetts

\footnotetext{
Abbreviations: 3-D = three-dimensional, abd-add = abductionadduction, ARMin = arm therapy robot, CADEN = cableactuated dexterous exoskeleton for neurorehabilitation, CCD = charge-coupled display, DOF = degree of freedom, EMG = electromyography, flx-ext = flexion-extension, ITRI = Industrial Technology Research Institute, L-Exos = light exoskeleton, PMA = pneumatic muscle actuator, SLERT = spring-loaded exoskeleton for resistance training, T-WREX = Therapy Wilmington Robotic Exoskeleton.

*Address all correspondence to Dar-Zen Chen, PhD; Department of Mechanical Engineering, National Taiwan University, No. 1, Sec. 4, Roosevelt Rd, Taipei, 0617 Taiwan (R.O.C.); +886-2-3366-2723; fax: +886-2-2369-2178.

Email: dzchen@ntu.edu.tw http://dx.doi.org/10.1682/JRRD.2012.12.0227
} 
Institute of Technology in the 1990s for use by muscular dystrophy patients. Numerous robotic devices that provide various forms of mechanical input to actively assist, resist, perturb, or increase the range of motion of the patient have been developed for hemiparetic upper-limb training. These devices can be broadly classified in two groups: end-effector robots and exoskeletons. MIT-Manus is an example of the former. One example of the latter is the arm therapy robot (ARMin). The ARMin III robot provides six actuated degrees of freedom (DOFs) to assist patients with shoulder, elbow, and wrist movements [3]. The MarylandGeorgetown-Army Exoskeleton is composed of a serial assemblage of five powered linkages and joints, allowing for full therapeutic exercise of the shoulder complex [4]. The light exoskeleton (L-Exos) is a five DOFs force-feedback exoskeleton, four of which are actuated and sensorized (three for the shoulder, one for the elbow), and the wrist is only sensorized [5]. Similar to L-Exos, the cableactuated dexterous exoskeleton for neurorehabilitation (CADEN)-7 is another cable-driven exoskeleton with an additional joint on the wrist mechanism, allowing wider range of motion [6]. Rigid links and motor-driven joints are common features of these designs [3-6], which add moving inertia to the segments of the human arm. L-Exos and CADEN-7 have attempted to lessen the increased inertia by placing the electric motors on the stationary base while driving by means of steel cables and reduction gears or pulleys integrated at the joint axis. Some designs $[3,6]$ added an actuated DOF to the design, allowing for shoulder girdle elevation and depression. Robotic-assisted upper-extremity repetitive therapy, known as RUPERT, has five actuated DOFs driven by compliant and safe pneumatic muscle actuators (PMAs) for assisting rehabilitation of arm function [7]. PMAs have high power-to-weight ratio over electric motors, which makes the exoskeleton compliant and inherently safer to wear. However, PMAs are more difficult to control because of nonlinear actuation characteristic. The cable-driven arm exoskeleton does not have rigid links and joints and does not restrict the natural DOFs of the human arm. Because human segment lengths are the only parameters in the kinematic model that determine how cable tensions result in torques at human joints, mechanical adjustments of segment lengths are not required, which is in contrast with a traditional exoskeleton [8]. Although some provide an active mode function [3,6] (i.e., the robot follows the arm of the user without disturbing his/her natural motion), unpowered passive arm exoskeletons are less costly, safer, and more compact than the actuated upperlimb robots described and may be more appropriate during the return to function phase and for home use. The Therapy Wilmington Robotic Exoskeleton (T-WREX), and its commercialized version Armeo Spring (Hocoma AG; Volketswil, Switzerland), is designed to enable individuals with arm weakness to achieve intense movement training without a supervising therapist. It is a passive, five DOFs, body-powered device that contains no robotic actuators, and it is a good example of such a device [9]. A recent study confirmed that the effectiveness of the robot is due to the large number of repetitions it provides; the robot's effect was similar to that of intensive therapist-provided therapy and was greater than the effect of usual care. The average cost of therapy per patient was the same for intensive therapist-provided therapy and robot therapy [10].

The studies cited previously indicate that the medical advances resulting from research on stroke and the resources invested in the rehabilitation of stroke victims have been focused on the acute and subacute recovery phases and that less attention has been directed at the more chronic recovery phases [3-9]. This situation has resulted in substantial health disparities in the later phases of stroke care. These circumstances clearly reflect the state of the science. There is a need for robotic devices that can be used to support rehabilitation in the later or more chronic phases of stroke care, in chronic diseases, and in other conditions to which the technology can be extended. Resistance training has recently been shown to also play a significant role in improving many health factors associated with the prevention of chronic diseases, including cardiovascular diseases, diabetes, hypertension, and obesity. The inclusion of resistance training as part of a physical exercise program has been endorsed by the American College of Sports Medicine, American Heart Association, and American Diabetes Association as an integral part of an overall health and fitness program. Furthermore, resistance training is recognized as a safe and effective strategy to enhance the neuromuscular systems of older adults and to improve muscle strength, power, and the ability to perform functional tasks, which may contribute to the prevention of falls and the maintenance of independence [11-15]. Free weights (such as dumbbells and barbells) and weight machines are the most familiar forms of resistance that may be used for muscle loading. The user's needs or patient's disability level will generally influence the type of resistance chosen. Concerns have been raised over the negative effects and the safety of resistance exercise as a physical therapy intervention and over the usage of the relevant equipment. Resistance equipment was originally 
designed to be used by nondisabled people, such as athletes or body builders, who are healthy enough to improve athletic performance or body fitness. Resistance equipment is usually used for gym- or studio-based exercise. In fact, only 23 percent of the U.S. population engages in leisure time resistance exercise activity at least two times a week, and participation rates are likely to be as low as 12.4 percent for individuals over the age of 65 [16-17]. These findings underscore the need for programs and more appropriate exercise devices that encourage nondisabled people and older adults to incorporate strength training into their lives along with regular physical activity.

The purpose of this study was to verify our hypothesis that, given zero-free-length springs, a spring-loaded upper-limb exoskeleton is capable of reducing the unfavorable lengthening of the muscles from inertial force during high-intensity free-weight exercises via investigating the joint torques exerted by either the spring-loaded exoskeleton for resistance training (SLERT) [18-19] or a dumbbell through the collection of experimental data, kinematics, and dynamic analysis data. This article extends the results of our previous static and dynamic studies [18-19]. The fundamental contributions of this article are as follows: (1) well-established and wellplanned experiments were conducted, analyzed, and interpreted; (2) zero-free-length spring design influenced exoskeleton performance as compared with free-weight exercise; (3) the feasibility of a spring-loaded upper-limb resistance training exoskeleton and its underlying design issues were tested. Experimental results demonstrated the effectiveness of the compliant and passive actuation of our upper-limb exoskeleton for resistance training.

\section{METHODS AND INSTRUMENTATION}

\section{Materials and Methods}

The SLERT is the revised version of our previous design [18], which was constructed to conduct a functional and performance-based evaluation of resistance training. The mechanical structure of the new prototype (Figure 1(a)) consists of four links that create four revolute joints covering the basic DOFs of the human arm, allowing the user to perform a shoulder abduction-adduction (abd-add), a flexion-extension (flx-ext), a horizontal flx-ext, and an elbow flx-ext motion, as well as three sets of zero-freelength springs that provide the required resistance for the designated upper-limb resistance training. Compared with the previous version of this exoskeleton [18-19], several design changes have been made to improve systemic functions. The location of the $z_{1}{ }^{*}$ axis was redesigned to shorten the distance between the $z_{1}$ axis and the $z_{1}{ }^{*}$ axis, which improves shoulder abd-add motion compliance. The arrangement of the $K_{3}$ spring has been moved from in front of link 2 to behind link 2 to avoid hindering the user's motion. In addition, the spring connection locations $A_{1}, A_{2}$, and $A_{3}$ were equipped with bearings and guiding slots for smoother motion when adjusting the spring connection locations. The device has also become more compact and lightweight. The design specifications of SLERT are given in Table 1.

The configuration of three revolute joints for the 3-DOF shoulder joint (axes $z_{0}, z_{1}$, and $z_{2}$ ) is illustrated in Figure 2(a). The revolute joints are constructed with ball bearings or thrust bearings to eliminate friction and to decrease defects of clearance. The 1-DOF elbow joint (axis $z_{3}$ ) is accommodated through a revolute joint, which adjusts the length of the upper limb. Thrust bearings are used to achieve the elbow flx-ext motion. The length of the forearm link can also be adjusted such that the device would fit different individuals, as shown in Figure 2(b). Both shoulder and elbow joints can be locked independently to perform the designated movements. The SLERT structure is constructed primarily from aluminum alloy materials with the high stress joint sections, links 3 and 4 , fabricated in steel.

A standard spring with a wire and pulley construction is used to emulate a zero-free-length spring, in which the force is proportional to the length of the spring rather than to its elongation, allowing the SLERT to increase or decrease the resistance by adjusting the spring length. All pulleys are equipped with bearings to reduce their friction and smooth their motion. The standard spring $K_{1}$ is fixed by a pin $B$ and connected to points $B_{1}$ (on link 1 ) and $A_{1}$ (on link 2) with wire and pulleys, as shown in Figure 2(a). The arrangement for springs $K_{2}$ and $K_{3}$ is the same as that for spring $K_{1}$, as is shown in Figure 1. To increase the intensity of the resistance exercise, the spring connection locations $A_{1}, A_{2}$, and $A_{3}$, which are separately integrated with nuts on the slide screws installed at link 2, can be adjusted using three slide screws. The required resistance in our design is generated by adjusting the connecting locations $\left(A_{1}, A_{2}\right.$, and $\left.A_{3}\right)$ of the springs rather than by changing the stiffness of the springs. This characteristic provides more convenience and flexibility in carrying out the resistance exercise program. 
(a)

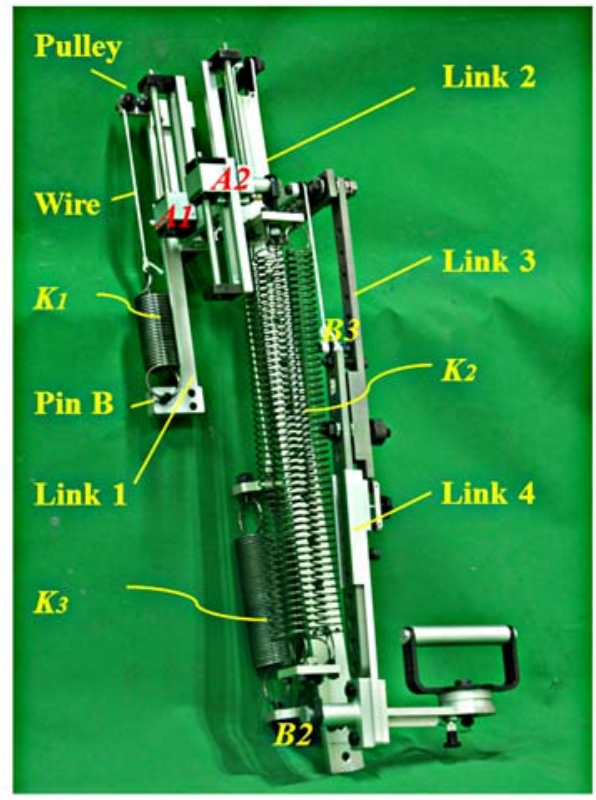

(b)

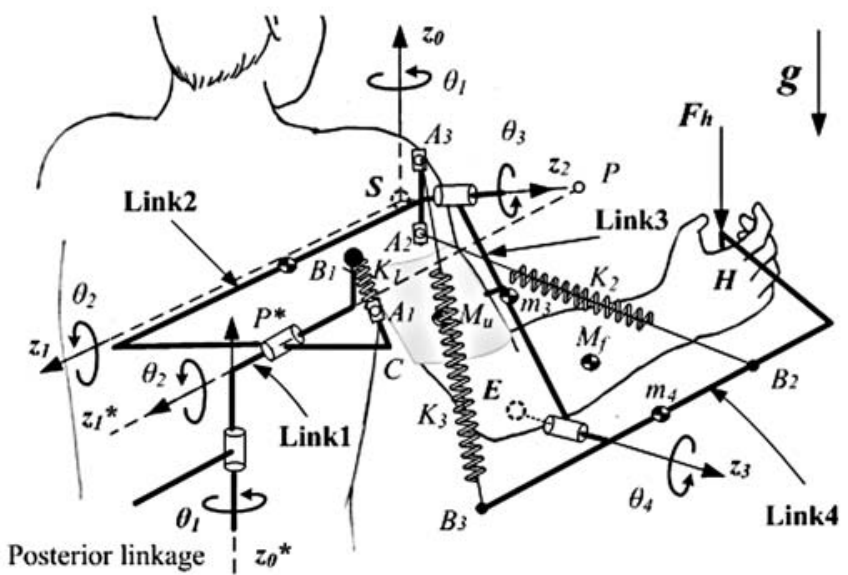

Figure 1.

New prototype and kinematic diagram. (a) Perspective view of spring-loaded exoskeleton for resistance training (SLERT). (b) Kinematic diagram of SLERT. $\theta_{1}=$ rotational angle for shoulder horizontal abduction-adduction, $\theta_{2}=$ rotational angle for shoulder abduction-adduction, $\theta_{3}=$ rotational angle for shoulder flexion-extension, $\theta_{4}=$ rotational angle for elbow flexion-extension, $A=$ connection point, $B=$ connection point, $E=$ elbow joint, $F_{\mathrm{h}}=$ external load, $H=$ middle of palm of hand, $K=$ spring, $m_{3}=$ mass of link $3, m_{4}=$ mass of link $4, M_{\mathrm{f}}=$ center of mass of forearm, $M_{\mathrm{u}}=$ center of mass of upper arm, $P=$ intersection of axes $z_{1}{ }^{*}$ and $z_{2}, P^{*}=$ intersection of link 1 and link 2, $S$ = glenohumeral joint.

Table 1.

Design specifications of spring-loaded exoskeleton for resistance training.

\begin{tabular}{lc}
\hline \multicolumn{1}{c}{ Property } & Target value \\
\hline Resistance (kg) & $1-5$ \\
Upper-Arm Length (mm) & $224-286$ \\
Forearm Length (mm) & $267-368$ \\
Range of Motion & \\
$\quad$ Shoulder abd-add $\left(^{\circ}\right)$ & $0-180$ \\
Shoulder flx-ext $\left(^{\circ}\right)$ & $0-180$ \\
Elbow flx-ext $\left(^{\circ}\right)$ & $0-145$ \\
Spring Stiffness & \\
$K_{1}(\mathrm{kgw} / \mathrm{mm})$ & 0.15 \\
$K_{2}(\mathrm{kgw} / \mathrm{mm})$ & 0.05 \\
$K_{3}(\mathrm{kgw} / \mathrm{mm})$ & 0.07 \\
\hline abd-add $=$ abduction-adduction, flx-ext $=$ flexion-extension. \\
\hline
\end{tabular}

Based on consideration of the target users and the purposes of their usage, the maximum resistant force of the current prototype is designed to be $49 \mathrm{~N}$ (corresponding to a $5 \mathrm{~kg}$ dumbbell). Based on the limitations and the mass properties of the linkages, the anthropometric parameters of humans, and the previous practical implementation experiences of this design, springs with a suitable stiffness were decided from the spring design constraints and chosen from a catalog of standard springs [20]. The design specifications are listed in Table 1.

Although the electrophysiology activation of a muscle and mechanical force generation can be observed as surface electromyography (EMG) and reflects the level of activation, the direct measures of muscle force are difficult because an EMG signal does not necessarily reflect the total amount of force or torque a muscle can produce, and some muscles inside the body are not accessible directly [21-22]. A motion-capture system was chosen to evaluate the performance of our design, and the joint torques were calculated by means of measuring kinematic data using a motion-capture system and compared our mechanism design to free-weight exercises while the subjects performed the designated movements. 


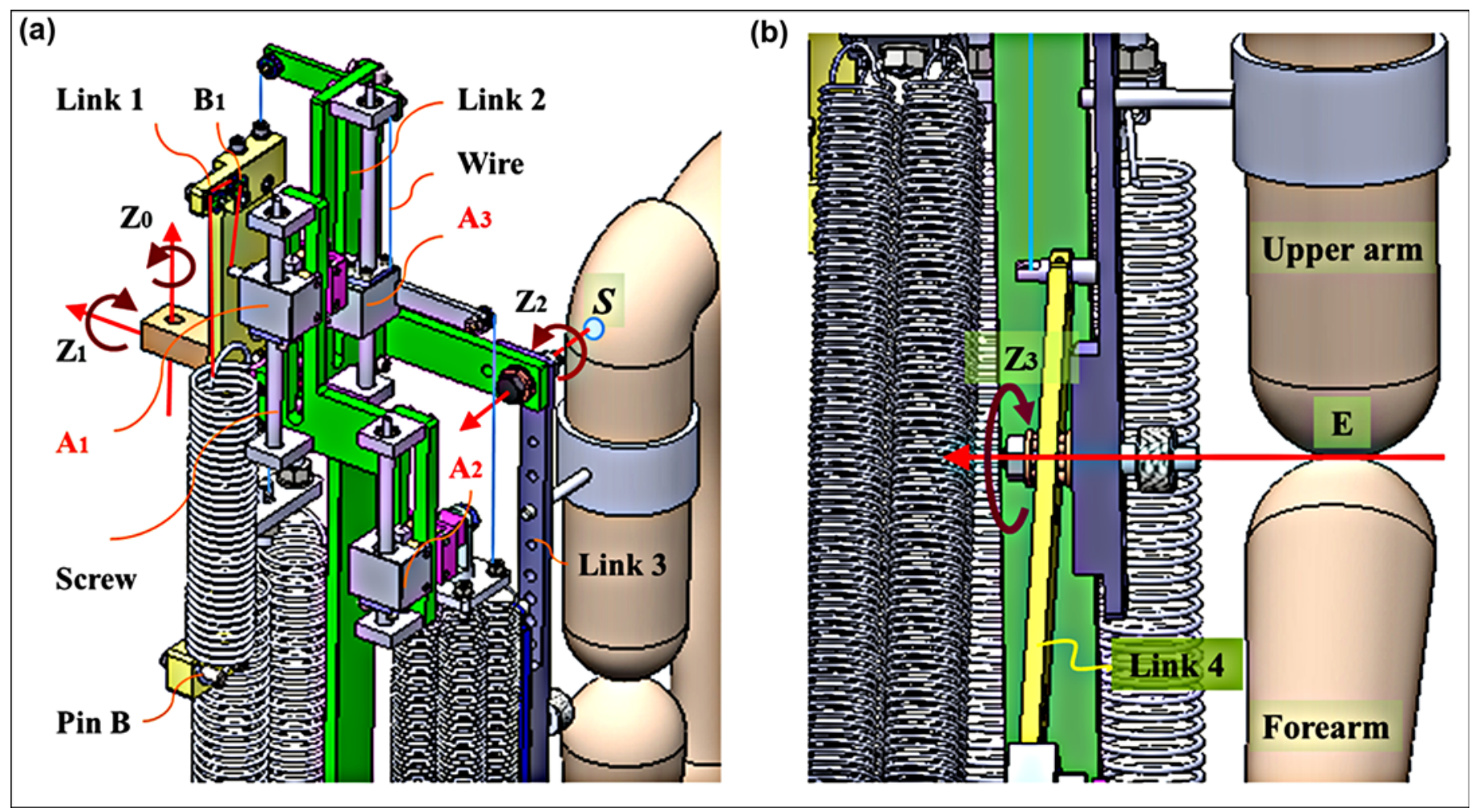

Figure 2.

Configuration computer-aided design drawings of shoulder and elbow joints of upper limb. (a) Configuration of shoulder joint exoskeleton. (b) Configuration of elbow joint. Shoulder joint comprises three revolute joint axes $z_{0}, z_{1}$, and $z_{2}$. $z_{3}$ is revolute joint axis of elbow joint (E). $A=$ connection point, $B=$ connection point, $S=$ glenohumeral joint.

\section{Study Subjects}

The device aimed to enable a patient or a nondisabled individual to move a limb at multiple joints in different planes for resistance training in a free and unconstrained environment. There are not significant characteristic differences in anthropometric parameters of young adults and older adults when calculating the joint torques. Considering the safety and potential risks of the test, young subjects were recruited for this initial evaluation. The inclusion of elderly subjects and larger sample sizes will occur after the system functionality of SLERT has been validated and the design of the system has been optimized.

Six nondisabled subjects (3 males and 3 females), referred to here as S1, S2, S3, S4, S5, and S6, volunteered to participate in this evaluation. Participants were required to have no previous shoulder or elbow pain or injury and no history of neural or musculoskeletal impairments. Each participant was required to read, understand, and sign an informed consent document that had been approved by the Industrial Technology
Research Institute's (ITRI's) ethics committee before instrumentation and data collection. The subjects' anthropometric parameters are listed in Table 2.

\section{Instrumentation}

The kinematic data of shoulder and elbow motions were acquired using a three-dimensional (3-D) passive optical motion capture system (Vicon MX, Vicon Motion Systems; Oxford, United Kingdom) at ITRI's research laboratory. This system consists of eight synchronized highspeed infrared charge-coupled display (CCD) cameras (Vicon MX-F20, Vicon Motion Systems) operating at a frame rate of $100 \mathrm{~Hz}$ to track eight reflective spherical markers measuring $14 \mathrm{~mm}$ in diameter and mounted by double-sided hypoallergenic tape on the subject in real time. The locations of the markers were predetermined bony anatomical landmarks, where subcutaneous tissue was thin and relatively fixed to the underlying skeleton, that were on the trunk and the upper limb of the subject, including the seventh cervical vertebra, clavicle, right shoulder 
Table 2.

Mean anthropometric parameters of subjects (S1-S6).

\begin{tabular}{|c|c|c|c|c|c|c|c|}
\hline Measure & All Subjects & S1 & $\mathrm{S} 2$ & S3 & S4 & S5 & S6 \\
\hline Age (yr) & $22.5 \pm 0.5$ & 22 & 23 & 23 & 23 & 22 & 22 \\
\hline Sex & $3 \mathrm{M} / 3 \mathrm{~F}$ & $\mathrm{M}$ & M & M & $\mathrm{F}$ & $\mathrm{F}$ & $\mathrm{F}$ \\
\hline Height (cm) & $164.8 \pm 6.0$ & 171 & 170 & 163 & 168 & 162 & 155 \\
\hline Upper-Arm Length (mm) & $276.6 \pm 20.9$ & 290 & 295 & 290 & 280 & 265 & 240 \\
\hline Forearm Length (mm) & $322.5 \pm 35.2$ & 352 & 355 & 340 & 320 & 310 & 260 \\
\hline
\end{tabular}

marker, right lateral elbow, right medial elbow, processus styloideus radius, processus styloideus ulna, and metacarpophalangeal joints of the right middle finger; these locations were used to define the segments that define position and orientation in 3-D space and were chosen for their minimal skin motion (Table 3). The markers were attached to anatomical landmarks found by palpation. All the markers on all subjects were attached by the same tester to remove interindividual variability as a source of error.

Before testing, an initial dynamic, or wand, calibration followed by a static, or calibration frame, calibration of the motion-capture system was performed to ensure the mean residual error was less than $1 \mathrm{~mm}$. It was necessary to ensure that each marker could be detected by at least two cameras at every instant during data recording and to ensure that no other shiny reflective materials on the subjects were detected by the cameras. While the subject was performing the selected free-weight exercise and shoulder abd-add, shoulder flx-ext, and elbow flx-ext movements with the spring-loaded upper-limb exoskeleton in the object-space in view of the CCD cameras, the motion analysis system recorded the movements of the upper-limb segments by tracking the 3-D position of the markers relative to a fixed laboratory coordinate frame. During testing, the spatial coordinates of the eight markers were captured, reconstructed, and labeled, and gaps were filled by the Nexus software (Nexus 1.3, Vicon Motion Systems).

The verification test consisted of shoulder abd-add, flx-ext, and elbow flx-ext movements of the right upper limb. The Vicon motion data were collected from both the male and female subjects.

\section{Experimental Protocols}

Two exercise sessions (free-weight exercises and upper-limb exoskeleton motion) were conducted for evaluation. Each session consisted of three resistance training exercises (shoulder abd-add, flx-ext, and elbow flx-ext) with six levels of difficulty (1, 3, and $5 \mathrm{~kg}$ resistance; 1 and $2 \mathrm{~s}$ motion speed) for the male subjects and four levels (1 and $3 \mathrm{~kg}$ resistance; 1 and $2 \mathrm{~s}$ motion speed) for the female subjects. Because joint torques vary as the resistance increases, step loads (1, 3, and 5) were chosen to allow us to conduct experiments at a reasonable scale. Because of sex differences in muscular strength, female subjects lifted only 1 and $3 \mathrm{~kg}$ resistances in the actual test. Each movement was performed in a slow, controlled manner: lifting ( 1 and 2 s) and lowering ( 1 and 2 s) without sudden jerks or acceleration for six consecutive repetitions. A metronome was employed to help the subjects maintain the tempo of their movements, and a maximum of 5 min rest was given

Table 3.

Marker names, locations, placement, and corresponding segments.

\begin{tabular}{llll}
\hline \multicolumn{1}{c}{ Marker } & \multicolumn{1}{c}{ Location } & \multicolumn{1}{c}{ Marker Placement } & Segment \\
\hline C7 & 7th cervical vertebra & Spinous process of 7th cervical vertebra & Thorax \\
CLAV & Clavicle & Jugular notch where clavicle meets sternum & Thorax \\
RSHO & Right shoulder marker & Place on top of acromio-clavicular joint & Scapula \\
RLEL & Right lateral elbow & Place on lateral epicondyle approximating right elbow joint axis & Humerus \\
RMEL & Right medial elbow & Place on medial epicondyle approximating right elbow joint axis \\
RMWR & Right wrist marker radius & Processus styloideus radius & Fumerus \\
RLWR & Right wrist marker ulna & Processus styloideus ulna & Forearm \\
RFIN & Right middle finger & Metacarpophalangeal joints of right middle finger & Hand \\
\hline \hline
\end{tabular}


between each set and exercise. Instructions on how to perform the motion were illustrated and demonstrated to the subjects at the beginning of the test. Subjects were also instructed to keep their left arms resting by their sides to ensure that they did not obscure or compensate for the motion of the right arm. The ranges of the movement evaluated and the grip patterns used are shown in Figure 3. The designated arm motions were included in the experimental protocol (Table 4). The dumbbell weights of 1, 3, and $5 \mathrm{~kg}$ and the equivalent resistances are set for free-weight exercise and upper-limb exoskeleton motion, respectively. If the subjects were obviously out of pace (too fast or too slow) or if mistakes were made, the recordings were discarded and the measurement was restarted. Warm-up trial practices were allowed before the actual tests to reduce the number of failed trials due to inaccurate pace or movement.

The starting position for the kinematic studies was defined as standing comfortably with the arms at the sides and the forearms naturally rotated in a relaxed posture (pronation). Every action and general motion started from an initial arm position in which the arm was fully extended along the body.

\section{Data Analysis}

Analyses were performed on the subjects' right shoulders and elbows. Joint torques at the shoulder and elbow were calculated via inverse dynamics. The analysis began with the most distal segment and continued upward through the kinematic chain, and the requirements of all the external forces acting on the system were known. The joint torques were then calculated by a 3-D generic inverse dynamic method [23]. Trial data from the exercises were captured, processed, and reviewed in the Vicon Nexus platform and postprocessed offline using MATLAB (MathWorks; Natick, Massachusetts). To suppress noise, the collected motion data were low-pass filtered by a fourth-order Butterworth filter at a cut-off frequency of $5 \mathrm{~Hz}$. Trial data over the required range of motion were also truncated. Of the 1,080 initial trials, only the first three data points of each of the 6 repeated trials (540 trials) were analyzed to obtain an average of (a)

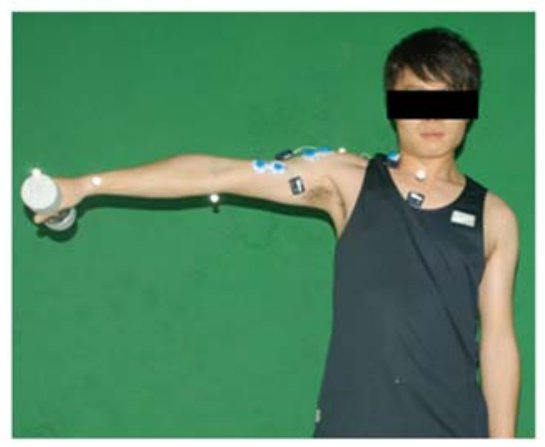

(d)

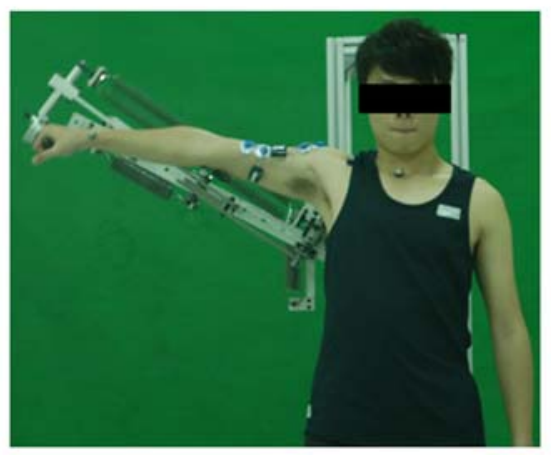

(b)

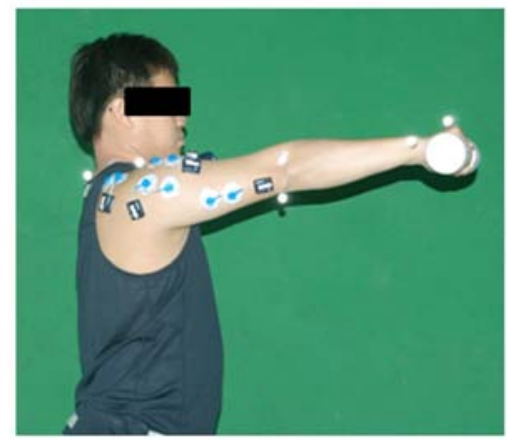

(e)

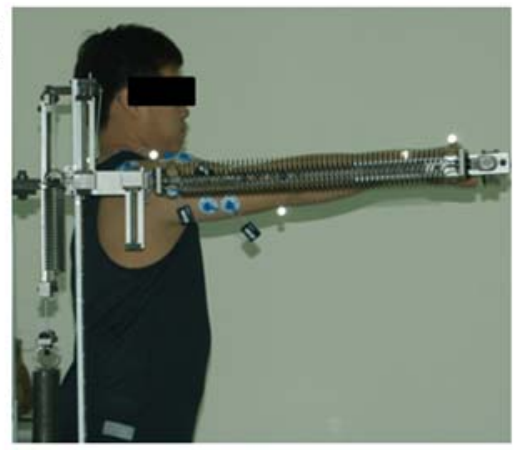

(c)

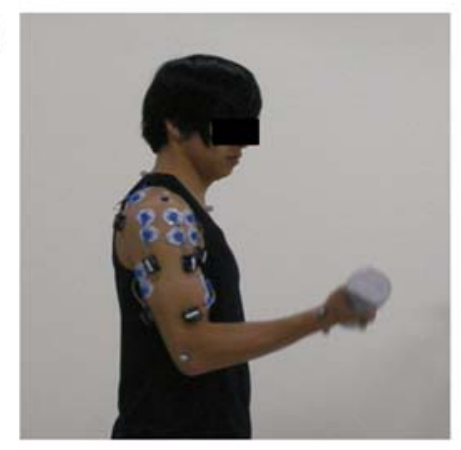

(f)

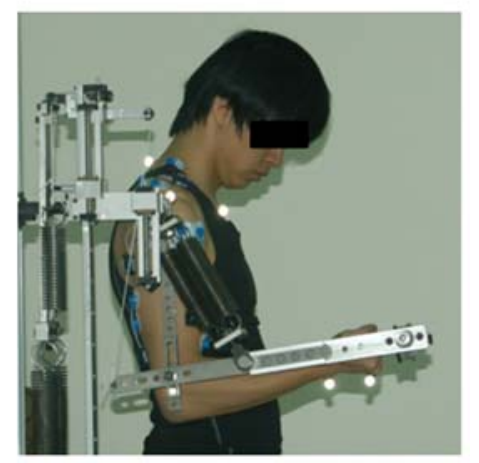

Figure 3.

Movement and grip patterns of free-weight exercises and exoskeleton motions. (a) Dumbbell lateral raise, (b) dumbbell frontal raise, (c) dumbbell curl, (d) shoulder abduction-adduction motion, (e) shoulder flexion-extension motion, and (f) elbow flexion-extension motion. 
JRRD, Volume 51, Number 1, 2014

Table 4.

Protocols for dumbbell movements and movements used with exoskeleton.

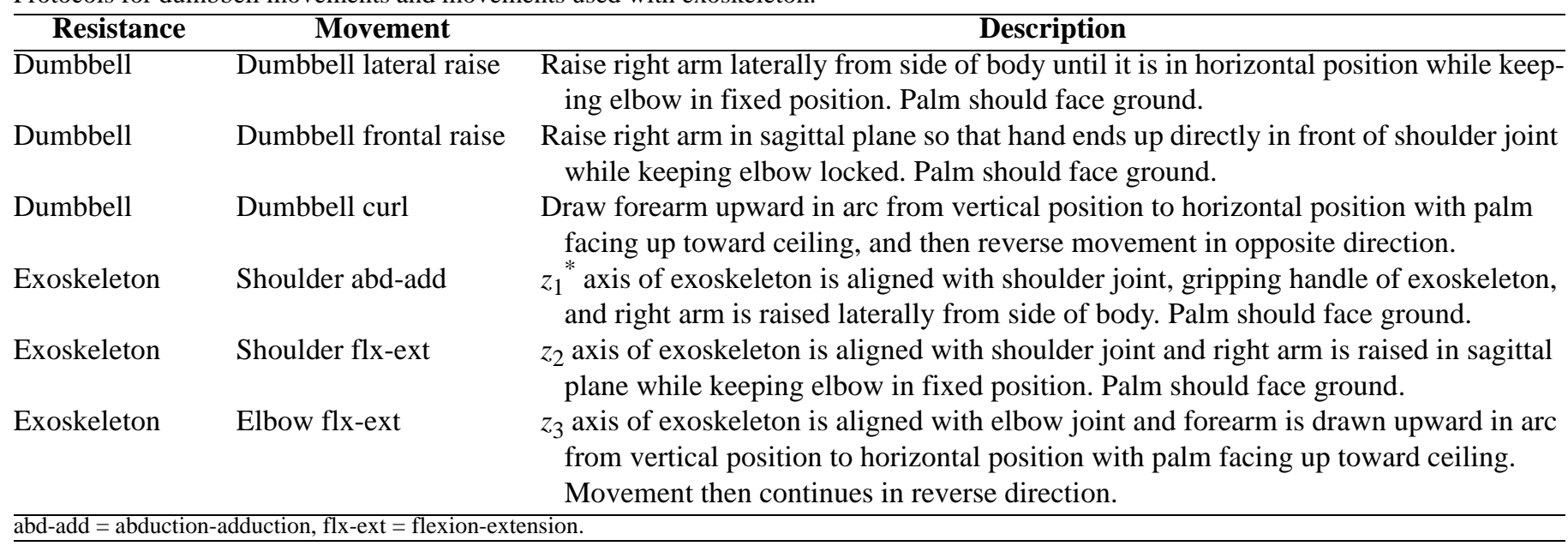

3 attempts. If one of these three data points was unfit for data analysis, the fourth, fifth, or sixth data points were analyzed (180 trials); therefore, a total of 720 trials were analyzed. The mean joint torques were then obtained from the mean value of the torques of the three male or three female subjects.

\section{RESULTS}

Figures $\mathbf{4}$ and $\mathbf{5}$ show the comparisons between the joint torques of free-weight exercises and the resistance exercise using the upper-limb exoskeleton for male and female subjects at two different motion speeds (velocity and inertia effects were neglected). In Figures 4(a-d)

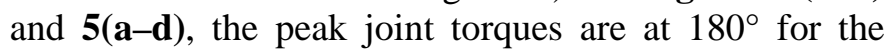
shoulder abd-add exercise and $90^{\circ}$ for the flx-ext exercise. The joints would generate higher torques when the upper limb straightens in the horizontal position, which is the point at which the moment arm is the farthest away from the resistant force while being perpendicular to the joint. A similar explanation can be applied to the elbow flx-ext exercise, as shown in Figures 4(e-f) and 5(e-f). The mean peak torques of the exercise at two different motion speeds, without considering the inertia effect, for male subjects, female subjects, and their differences are listed in Tables 5 and $\mathbf{6}$.

Figures 6 and $\mathbf{7}$ show comparisons between the joint torques of free-weight exercises and the resistance exercises using the upper-limb exoskeleton mechanism at two different motion speeds when considering the velocity and the effects of inertia. Generally speaking, dumbbell exercises generate a higher moment of inertia in the shoulder joint, and the inertial effect becomes more obvious as the resistance load or the velocity increases.

\section{DISCUSSION}

Figure 8 compares the mass moments of inertia calculated from the 1, 3, and $5 \mathrm{~kg}$ fixed-weight dumbbells and the exoskeleton motion of the prototype for the six subjects. The calculations indicate that holding the dumbbell at the distal end of the upper limb produces a larger inertial effect than the current upper-limb exoskeleton prototype; moreover, as the weight of the dumbbell increases, the inertial effect also increases dramatically. We found that link 2 had a larger mass moment of inertia in the current exoskeleton design relative to the $1 \mathrm{~kg}$ dumbbell, which agrees with the experimental results described in Figure 8(a-b). Therefore, the mass of the linkage should further conform to certain additional constraints with respect to the mass moment of inertia of the dumbbell and the linkage, which can be obtained from the dynamic analysis [19].

The experimental results indicate that the motion tendency of the shoulder abd-add, shoulder flx-ext, and elbow flx-ext motions for resistance exercises performed with the upper-limb exoskeleton are nearly equivalent to the joint torques obtained from the upper-limb dumbbell lateral raise, the dumbbell frontal raise, and the dumbbell curl according to the static joint torque analysis. However, the shoulder joint sustains a smaller moment of 
(a)

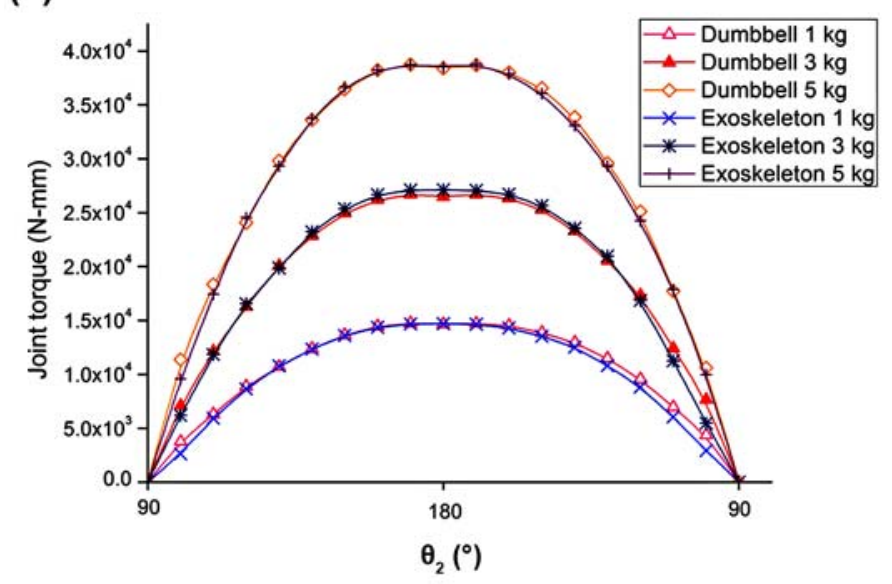

(c)

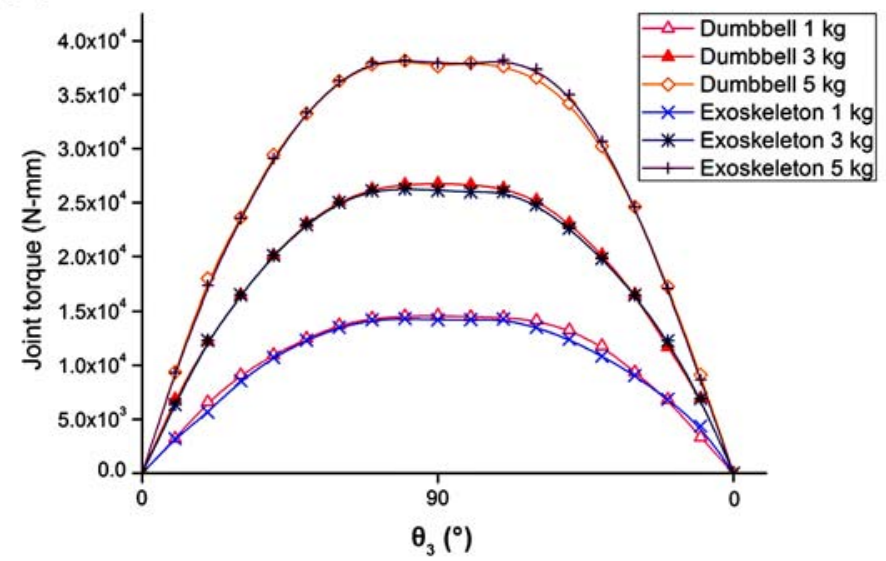

(e)

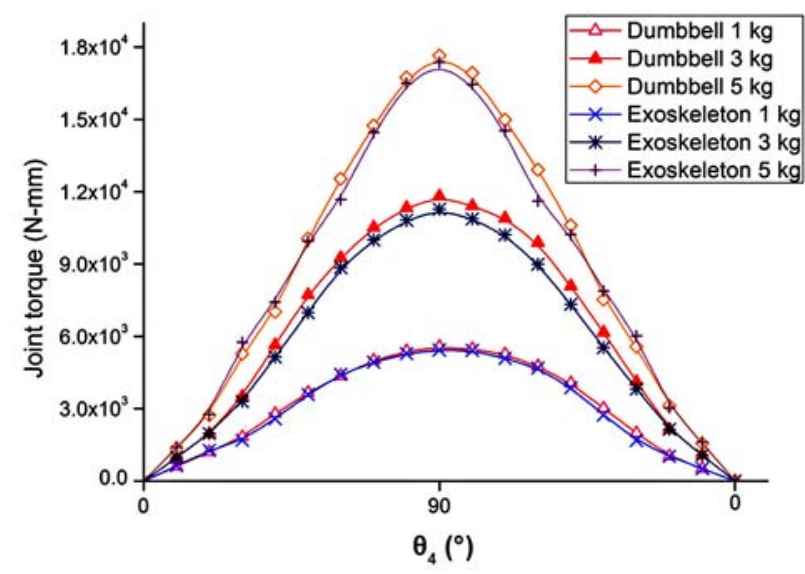

(b)

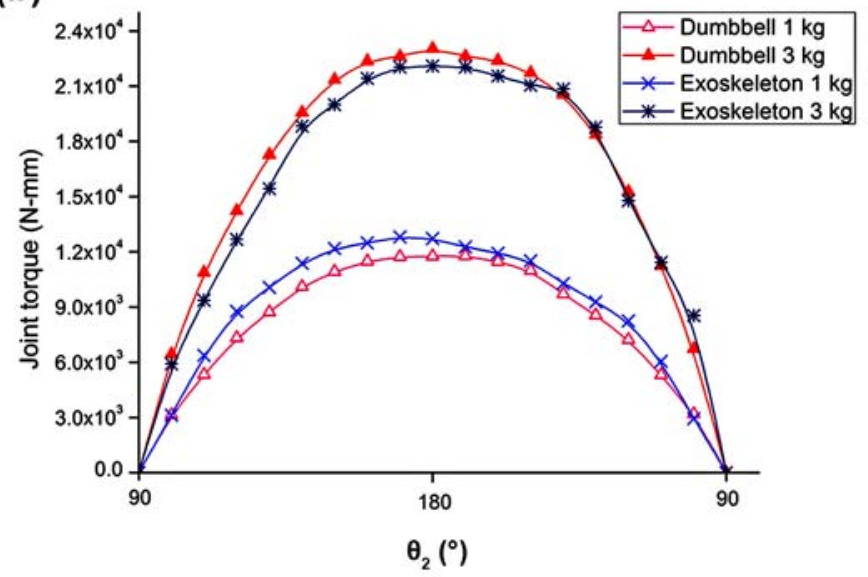

(d)

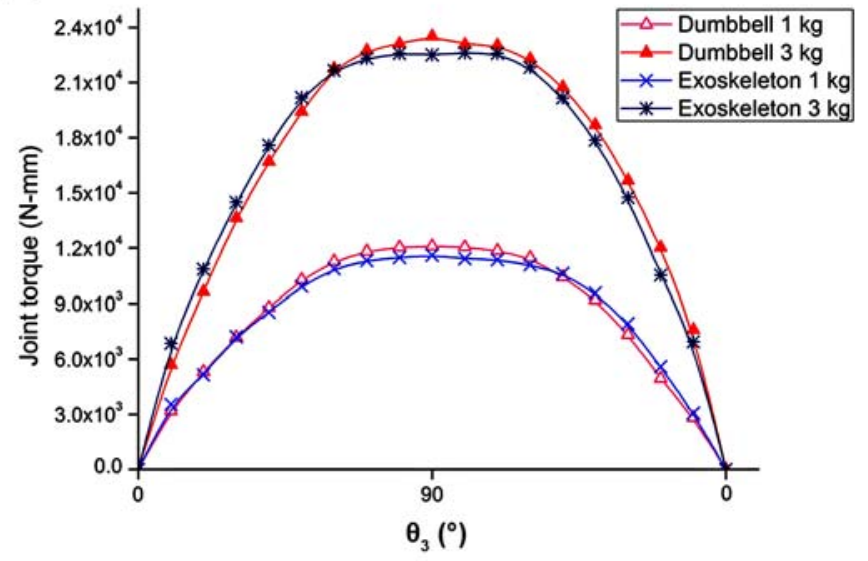

(f)

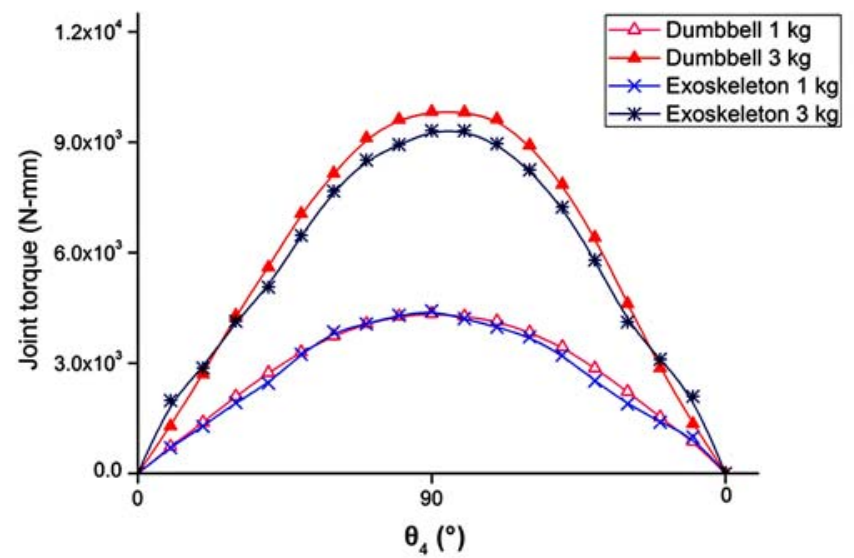

Figure 4.

Experimental joint torques of free-weight and resistance exercises using upper-limb exoskeleton without inertial effects (1 s lifting and $1 \mathrm{~s}$ lowering motion speed). (a) Shoulder abduction-adduction motion (male subjects). (b) Shoulder abduction-adduction motion (female subjects). (c) Shoulder flexion-extension motion (male subjects). (d) Shoulder flexion-extension motion (female subjects). (e) Elbow flexion-extension motion (male subjects). (f) Elbow flexion-extension motion (female subjects). 
(a)

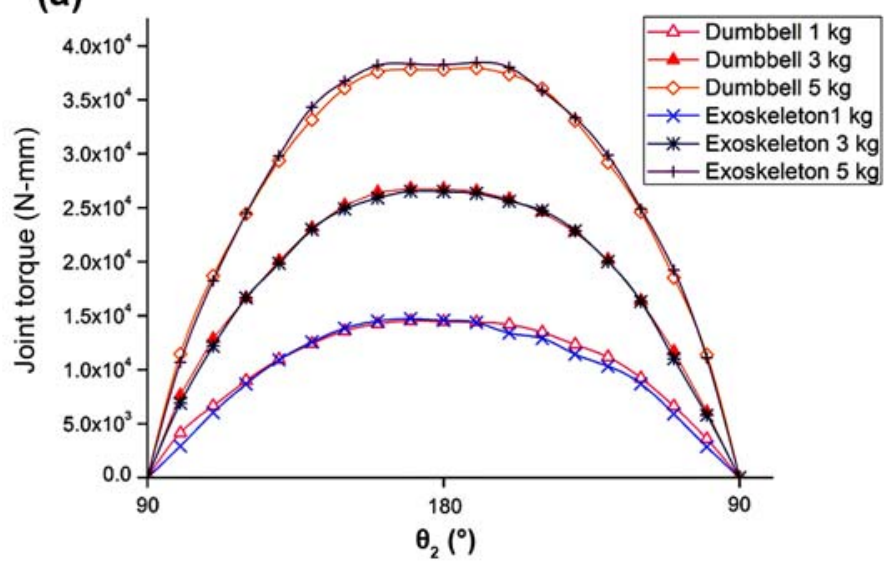

(c)

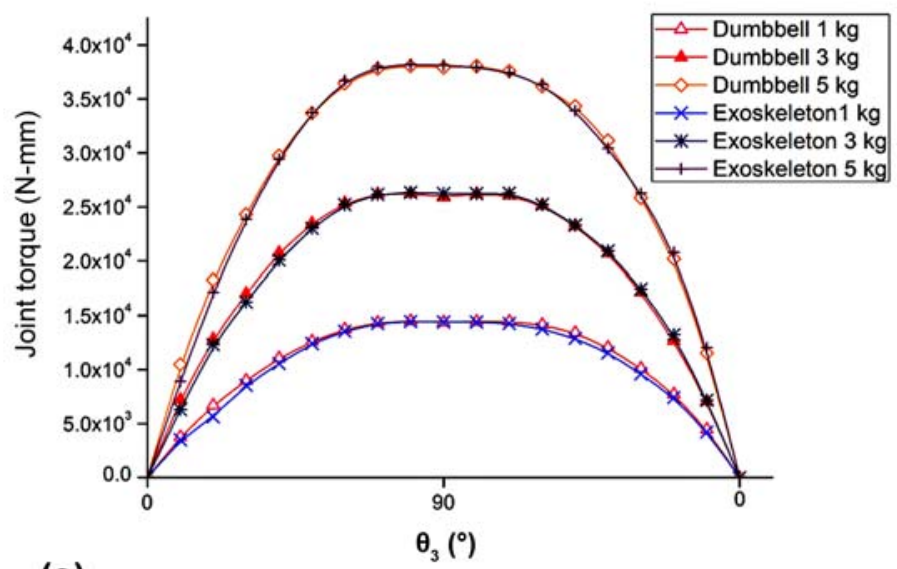

(e)

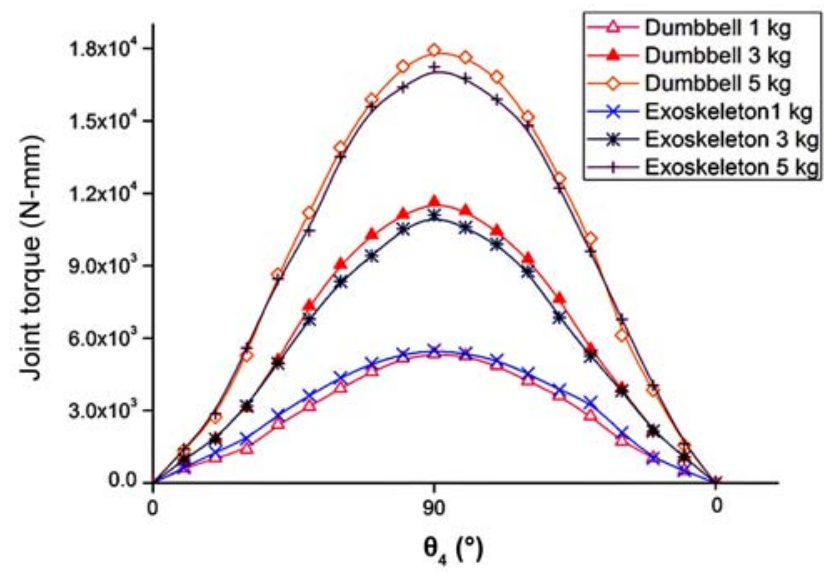

(b)

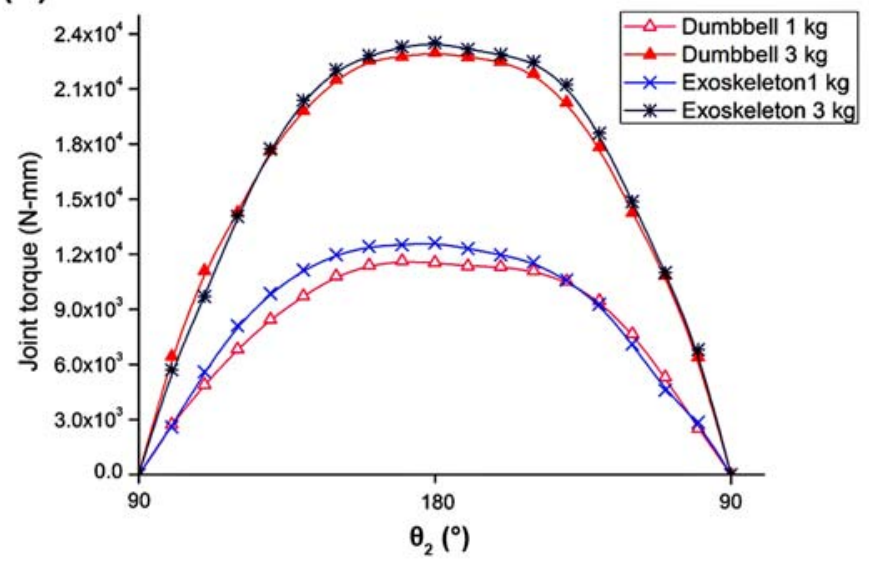

(d)

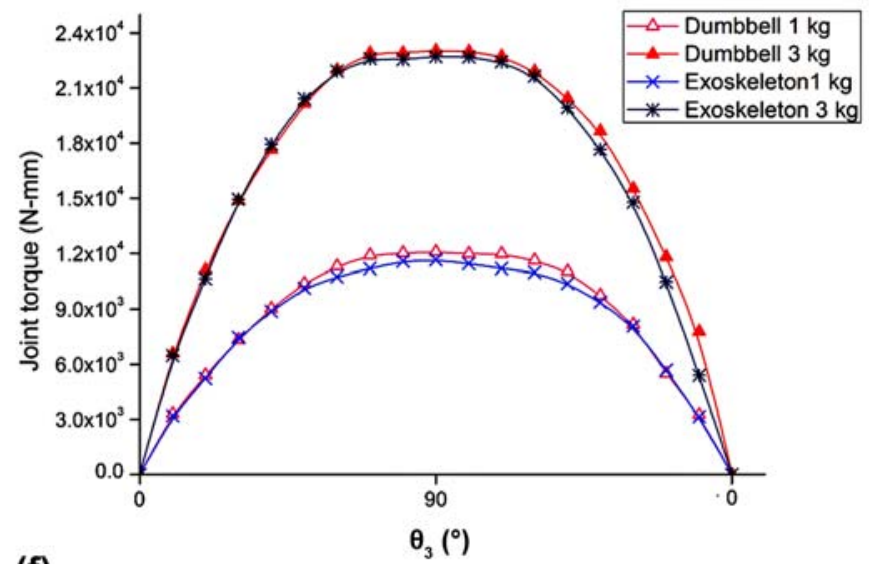

(f)

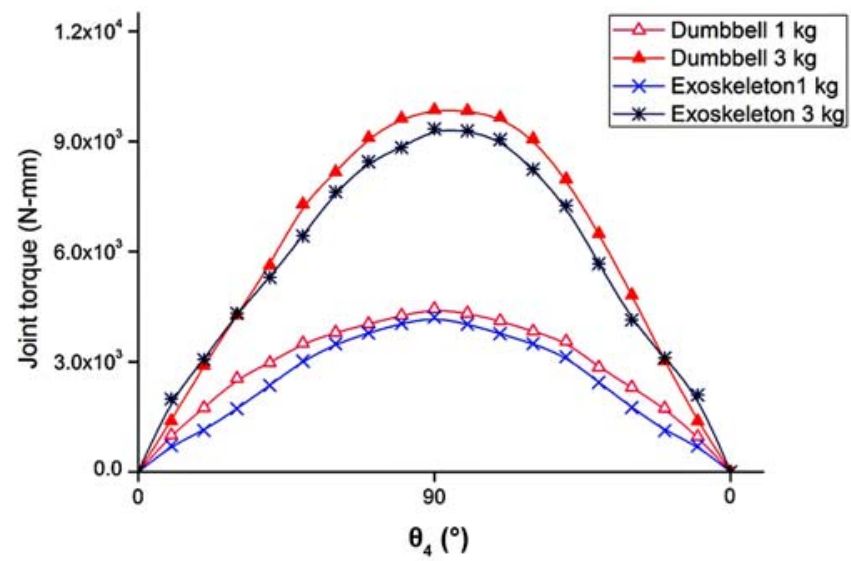

Figure 5.

Experimental joint torques of free-weight and resistance exercises using upper-limb exoskeleton without inertial effects ( $2 \mathrm{~s}$ lifting and $2 \mathrm{~s}$ lowering motion speed). (a) Shoulder abduction-adduction motion (male subjects). (b) Shoulder abduction-adduction motion (female subjects). (c) Shoulder flexion-extension motion (male subjects). (d) Shoulder flexion-extension motion (female subjects). (e) Elbow flexion-extension motion (male subjects). (f) Elbow flexion-extension motion (female subjects). 
Table 5.

Mean peak torques and differences for free-weight and upper-limb exoskeleton exercises at $1 \mathrm{~s}$ lifting and $1 \mathrm{~s}$ lowering motion speeds.

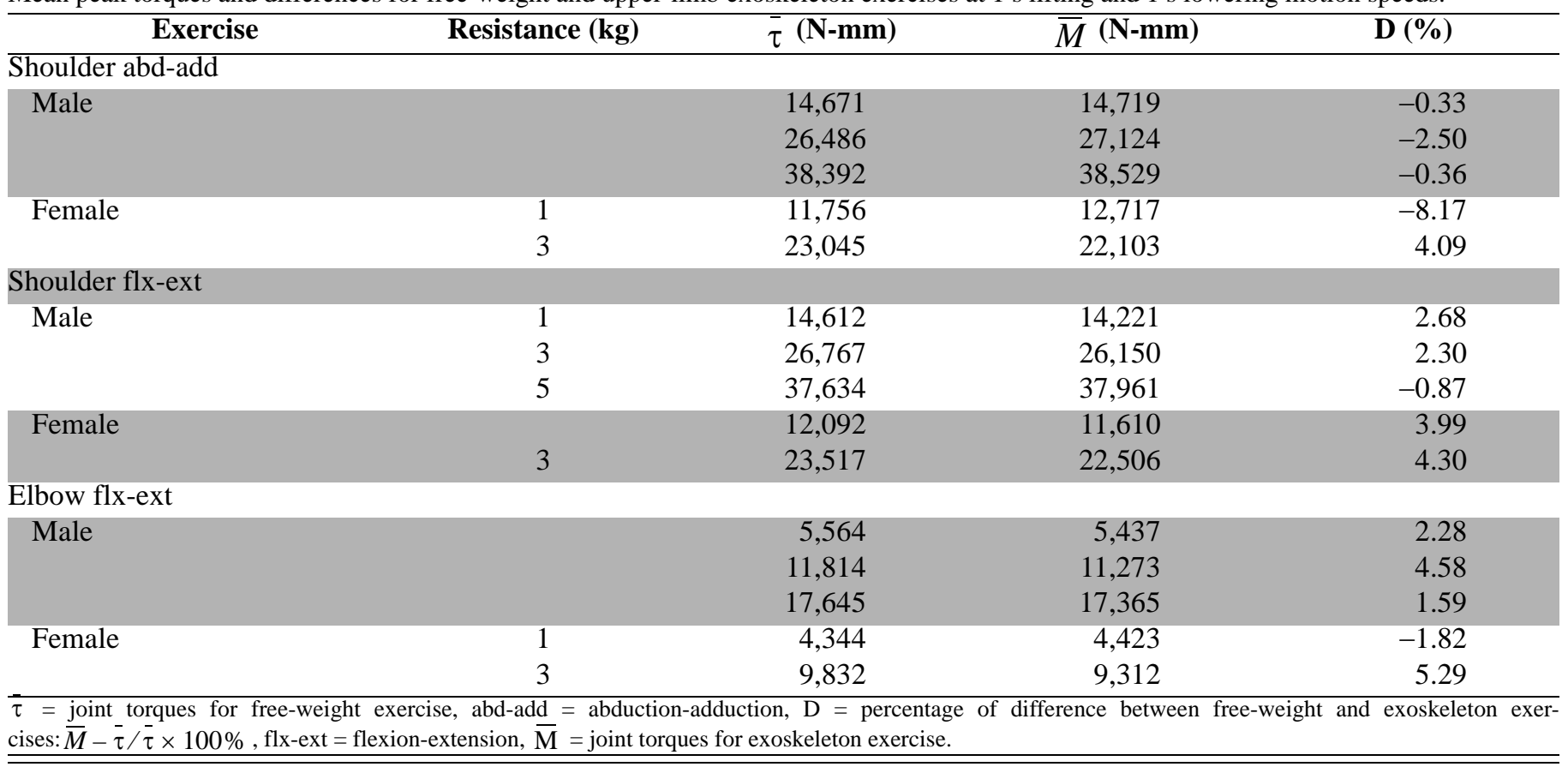

Table 6.

Mean peak torques and differences for free-weight and upper-limb exoskeleton exercises at $2 \mathrm{~s}$ lifting and $2 \mathrm{~s}$ lowering motion speeds.

\begin{tabular}{|c|c|c|c|c|}
\hline $\begin{array}{r}\text { Exercise } \\
\end{array}$ & Resistance (kg) & $\bar{\tau}(\mathbf{N}-\mathbf{m m})$ & $\bar{M}$ (N-mm) & D (\%) \\
\hline \multicolumn{5}{|l|}{ Shoulder abd-add } \\
\hline \multirow{2}{*}{ Male } & & 14,455 & 14,583 & -0.88 \\
\hline & 3 & 26,741 & 26,502 & 0.89 \\
\hline \multirow[t]{2}{*}{ Female } & 1 & 11,541 & 12,620 & -4.69 \\
\hline & 3 & 22,985 & 23,528 & -2.36 \\
\hline & 3 & 25,892 & 26,243 & -1.36 \\
\hline & 5 & 37,919 & 38,140 & -0.87 \\
\hline \multirow[t]{2}{*}{ Female } & & 12,081 & 11,657 & -0.58 \\
\hline & 3 & 23,043 & 22,719 & 1.41 \\
\hline \multicolumn{5}{|l|}{ Elbow flx-ext } \\
\hline \multirow[t]{2}{*}{ Female } & 1 & 4,437 & 4,214 & 5.02 \\
\hline & 3 & 9,858 & 9,340 & 5.25 \\
\hline
\end{tabular}

inertia when performing exercises using the upper-limb exoskeleton in the dynamic joint torque analysis. According to the results of the verification test, the drawbacks because of the effect of the mass moment of inertia in the free-weight exercises were clearly reduced by adding a small inertial spring to the design. 
(a)
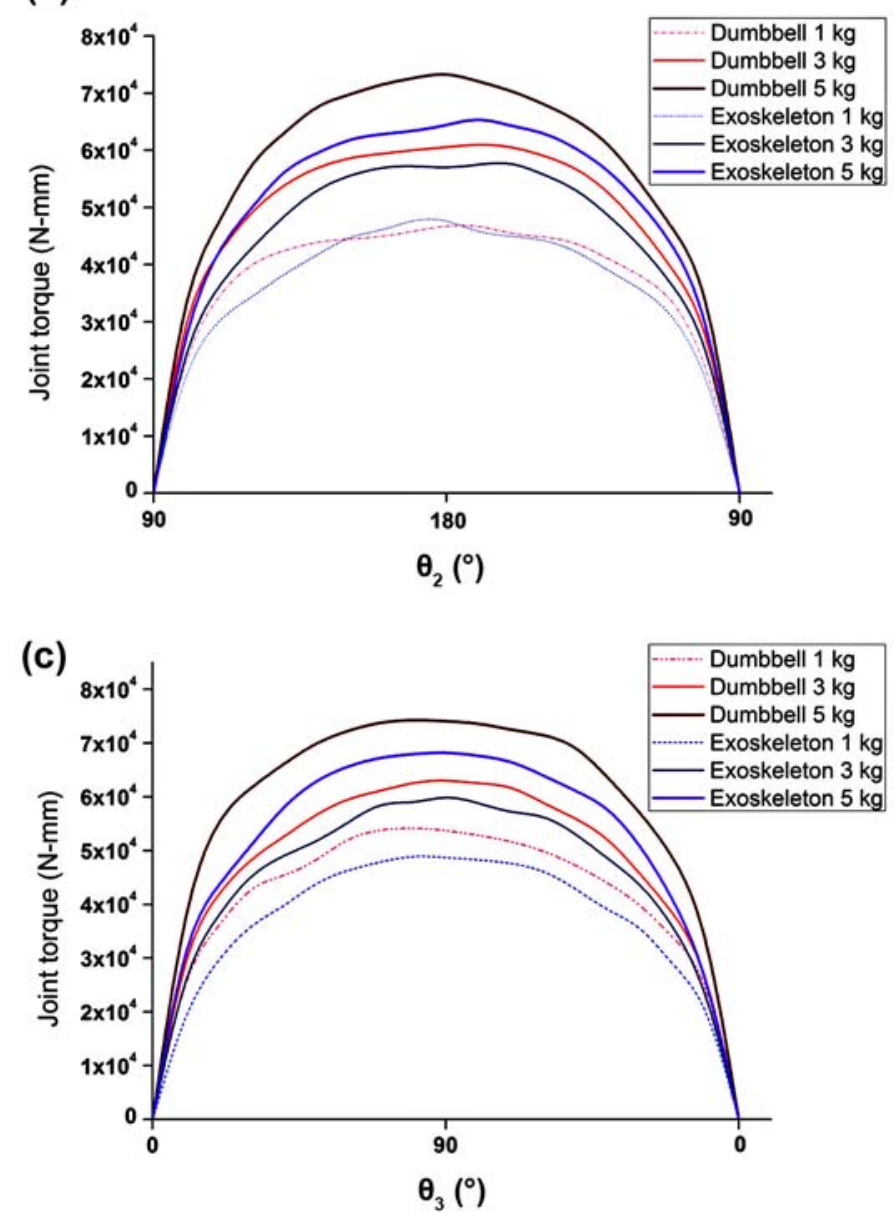

(e)

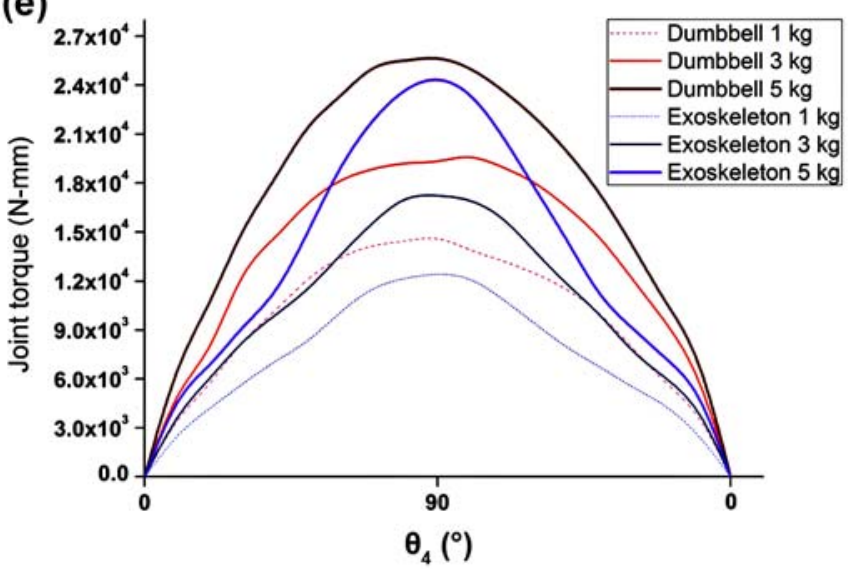

(b)
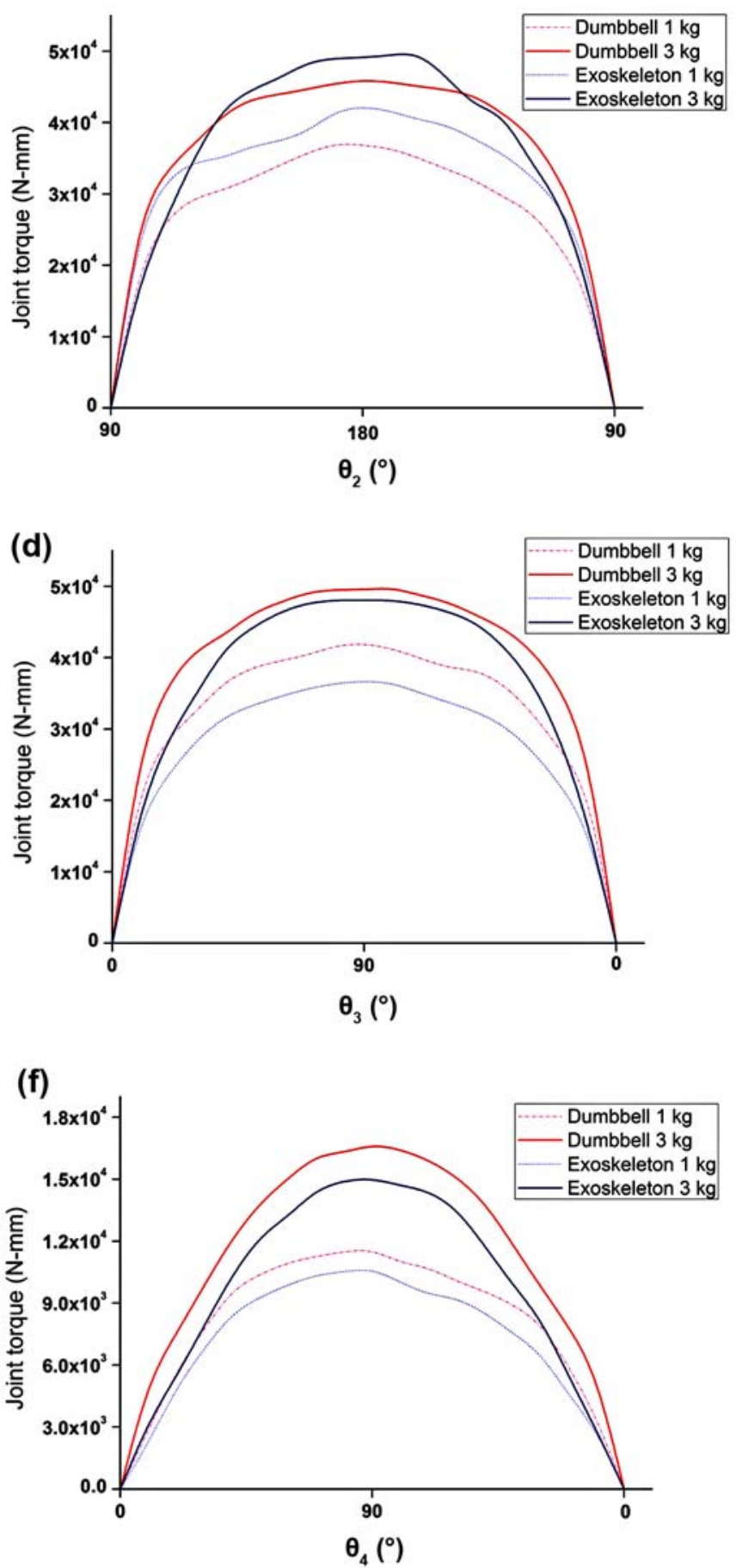

Figure 6.

Experimental joint torques of free-weight and resistance exercises using upper-limb exoskeleton with inertial effect (1 $\mathrm{s}$ lifting and $1 \mathrm{~s}$ lowering motion speed). (a) Shoulder abduction-adduction motion (male subjects). (b) Shoulder abduction-adduction motion (female subjects). (c) Shoulder flexion-extension motion (male subjects). (d) Shoulder flexion-extension motion (female subjects). (e) Elbow flexion-extension motion (male subjects). (f) Elbow flexion-extension motion (female subjects). 
(a)
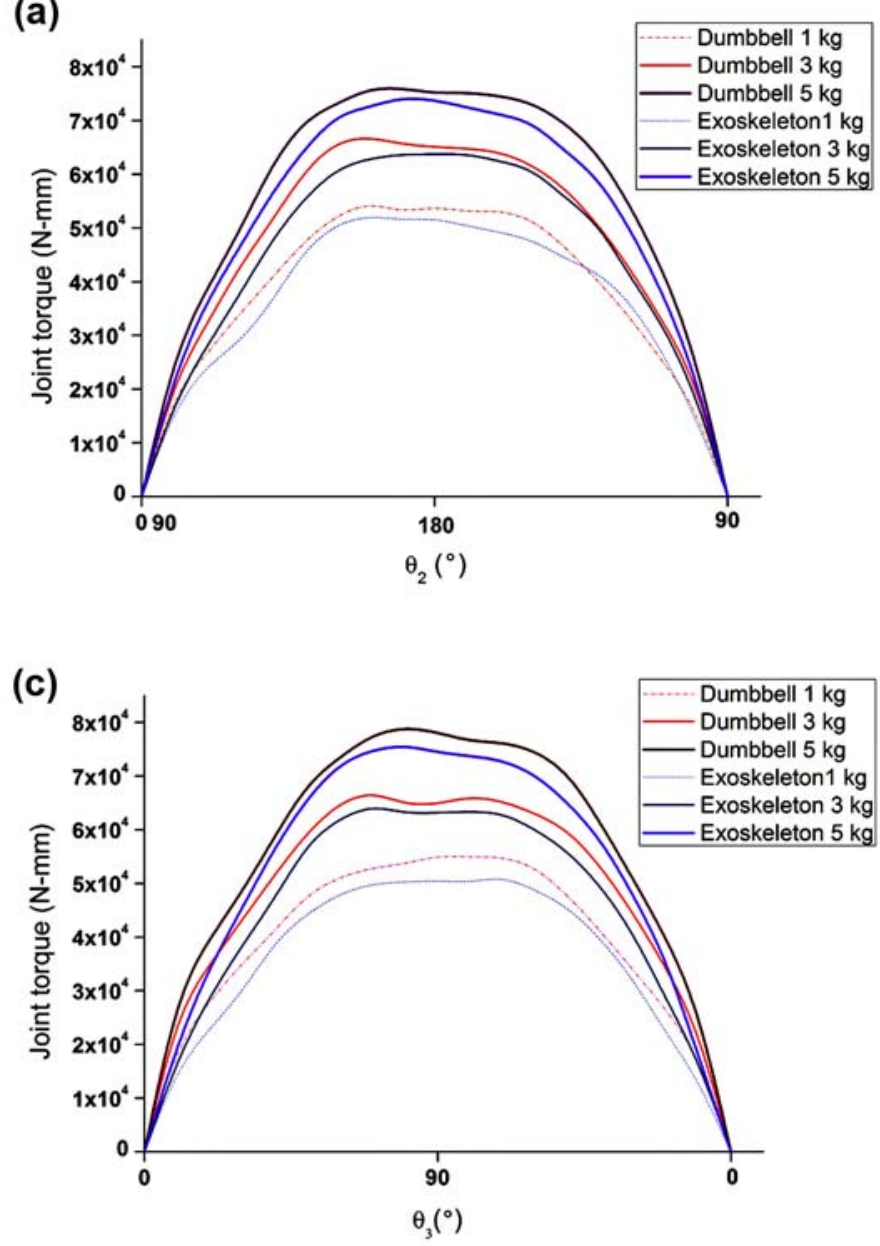

(e)

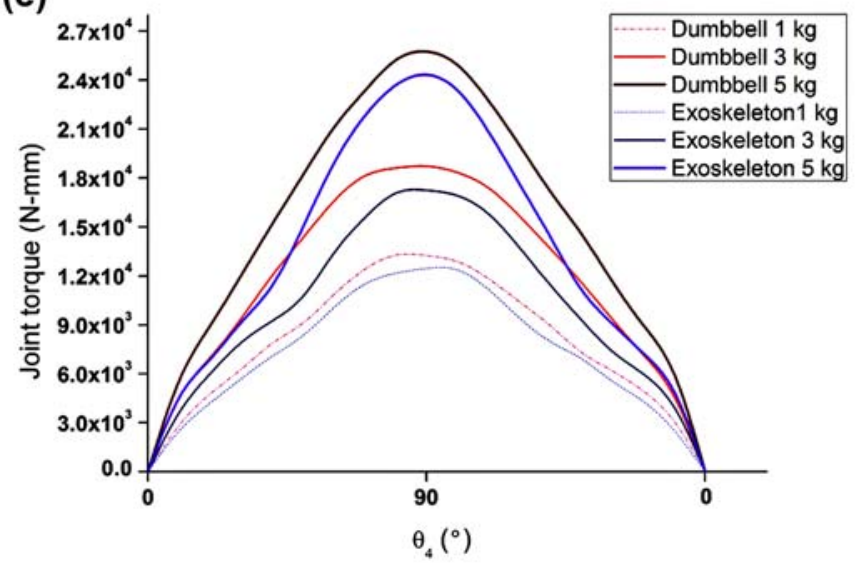

(b)
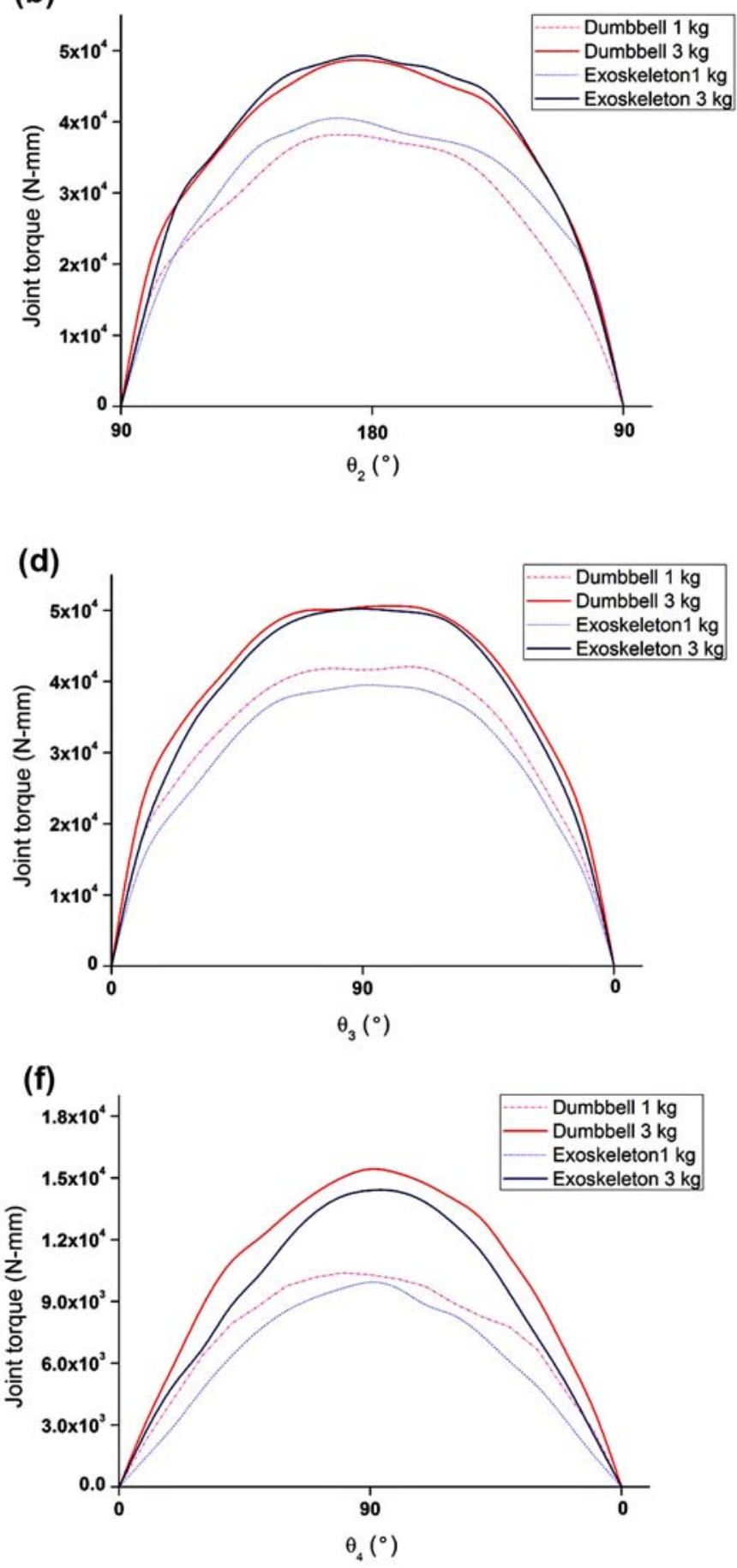

Figure 7.

Experimental joint torques of free-weight and resistance exercises using upper-limb exoskeleton with inertial effect $(2 \mathrm{~s}$ lifting and $2 \mathrm{~s}$ lowering motion speed). (a) Shoulder abduction-adduction motion (male subjects). (b) Shoulder abduction-adduction motion (female subjects). (c) Shoulder flexion-extension motion (male subjects). (d) Shoulder flexion-extension motion (female subjects). (e) Elbow flexion-extension motion (male subjects). (f) Elbow flexion-extension motion (female subjects). 
(a)

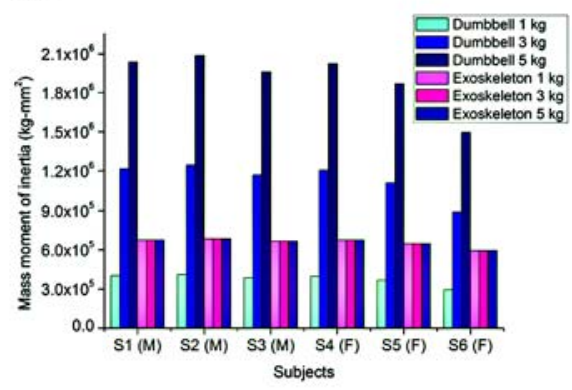

(b)

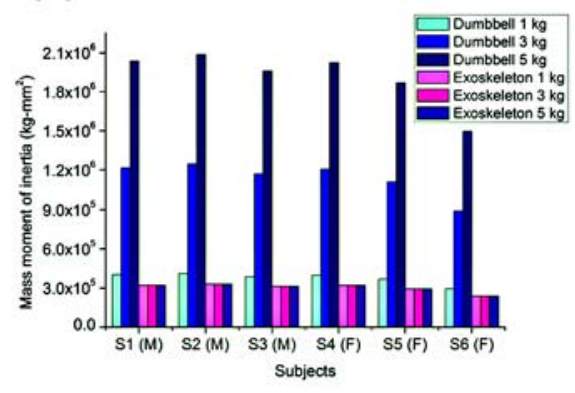

(c)

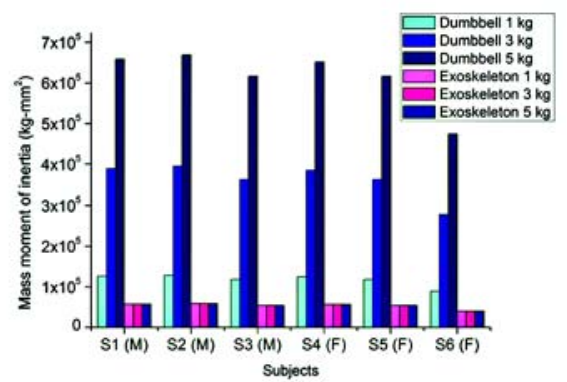

Figure 8.

Mass of moment of inertia effect caused by dumbbell or exoskeleton on subjects. (a) Shoulder abduction-adduction movement. (b) Shoulder flexion-extension movement. (c) Elbow flexion-extension movement. $F=$ female, $M=$ male.

Most of the rehabilitation and healthcare robotic exoskeletons mentioned in the introduction are primarily designed to actively assist patients with motions (except for the T-WREX) [3-9]. Design trade-offs are made among various kinematic designs, actuator/drive technologies, power-to-weight ratios, and control architectures. In contrast, most exercise training devices are bodypower oriented. Exercise training device users are expected to perform motions by themselves to gain strength, power, and endurance from the exercise. This study proposed a rehabilitation exercise training device designed from a strength exercise point of view to reduce the risk of adverse health outcomes, and we developed a general design scheme for the use of spring-loaded upper-limb exoskeletons for resistance training. Compared with our previous studies [18-19], efforts have been made through well-designed experimental investigations to better understand the physical significance of using a spring-loaded exoskeleton for upper-limb resistance training. The collected data, along with the kinematics and dynamics analyses that were performed in our previous studies, may further our understanding of the design requirements of a spring-loaded exoskeleton for upper-limb resistance training. As a result, alternative designs, inventions, and new applications can be developed more easily, systematically, and efficiently for the benefit of resistance or rehabilitation training.

Knowledge of the muscle activity and the recruitment of muscle groups during different resistance training is of great importance for the study of the utility of the device. Future studies could focus on neuromuscular functional retraining for the elderly user and on the procurement of larger sample sizes to provide convincing evidence of the effectiveness of the SLERT in obtaining significant and impactful results.

\section{CONCLUSIONS}

We modified and reconstructed a passive upper-limb exoskeleton prototype in accordance with previous studies and conducted an analysis of online motion to record designated movements performed using free weights and the upper-limb exoskeleton as the resistance by six subjects. The static joint torques exerted at the shoulder and elbow joints when using the exoskeleton were shown to be nearly equal to the objective static joint torques obtained from models of free-weight exercises and kinematics at lower and moderate motion speeds. The dynamic joint torques of the shoulder and elbow joints for movements performed with the exoskeleton were shown to be smaller than the objective dynamic joint torques obtained from models of free-weight exercises and kinetics, and the effect became more obvious as the resistance load and the motion velocity increased. Comparisons of all of the exercises were obtained with good conformity. We have demonstrated that the device used in this study is capable of reducing the unfavorable lengthening of muscles during high-intensity free-weight exercises or joint overload caused by large moments of inertia. Finally, this study provides verification test results for an upper-limb exoskeleton and determines the appropriateness of the motion analysis evaluation method and the optimal configuration of a spring-loaded upperlimb exoskeleton. 


\section{ACKNOWLEDGMENTS}

\section{Author Contributions:}

Study concept and design: D. Z. Chen, T. M. Wu.

Acquisition of data: T. M. Wu.

Analysis and interpretation of data: T. M. Wu, D. Z. Chen.

Drafting of manuscript: T. M. Wu.

Critical revision of manuscript for important intellectual content:

D. Z. Chen, T. M. Wu.

Statistical analysis: T. M. Wu.

Administrative, technical, or material support: D. Z. Chen.

Study supervision: D. Z. Chen.

Financial Disclosures: The authors have declared that no competing interests exist.

Funding/Support: This material was unfunded at the time of manuscript preparation.

Additional Contributions: Dr. Wu is now with ITRI Southern Region Campus, ITRI, Taiwan.

Institutional Review: Each participant was required to read, understand, and sign an informed consent document that had been approved by the ITRI's ethics committee before instrumentation and data collection.

Participant Follow-Up: The authors do not plan to inform participants of the publication of this article.

\section{REFERENCES}

1. Mackay J, Mensah GA, Mendis S. The atlas of heart disease and stroke. Geneva (Switzerland): World Health Organization; 2004.

2. Brochard S, Robertson J, Médée B, Rémy-Néris O. What’s new in new technologies for upper extremity rehabilitation? Curr Opin Neurol. 2010;23(6):683-87.

[PMID:20852420]

http://dx.doi.org/10.1097/WCO.0b013e32833f61ce

3. Nef T, Guidali M, Riener R. ARMin III-arm therapy exoskeleton with an ergonomic shoulder actuation. Appl Bio Biomech. 2009;6:127-42.

http://dx.doi.org/10.1080/11762320902840179

4. Carignan C, Liszka M, Roderick S. Design of an arm exoskeleton with scapula motion for shoulder rehabilitation. Proceedings of the 12th International Conference on Advanced Robotics; 2005 Jul 18-20; Seattle, WA. p. 524-31.

5. Frisoli A, Rocchi F, Marcheschi S, Dettori A, Salsedo F, Bergamasco M. A new force-feedback arm exoskeleton for haptic interaction in virtual environments. Proceedings of the 1st Joint Eurohaptics Conference and Symposium on Haptic Interfaces for Virtual Environment and Teleoperator Systems; 2005 Mar 18-20; Pisa, Italy.

6. Rosen J, Perry JC, Manning N, Burns S, Hannaford B. The human arm kinematics and dynamics during daily activitiesToward a 7 DOF upper limb powered exoskeleton. Proceedings of the 12th International Conference on Advanced Robotics; 2005 Jul 18-20; Seattle, WA. p. 532-39.
7. Balasubramanian S, Wei R, Perez M, Shepard B, Koeneman E, Koeneman J, He J. RUPERT: An exoskeleton robot for assisting rehabilitation of arm functions. Proceedings of the Virtual Rehabilitation Conference; 2008 Aug 25-27; Vancouver, Canada. p. 163-67.

8. Mao Y, Agrawal SK. Design of a cable-driven arm exoskeleton (CAREX) for neural rehabilitation. IEEE Trans Robot. 2012;28(4):922-31.

http://dx.doi.org/10.1109/TRO.2012.2189496

9. Housman SJ, Le V, Rahman T, Sanchez RJ, Reinkensmeyer DJ. Arm-training with T-WREX after chronic stroke: Preliminary results of a randomized controlled trial. Proceedings of the IEEE 10th International Conference on Rehabilitation Robotics 2007 Jun 13-15; Noordwijk, the Netherlands. p. 562-68.

10. Lo AC, Guarino PD, Richards LG, Haselkorn JK, Wittenberg GF, Federman DG, Ringer RJ, Wagner TH, Krebs HI, Volpe BT, Bever CT Jr, Bravata DM, Duncan PW, Corn BH, Maffucci AD, Nadeau SE, Conroy SS, Powell JM, Huang GD, Peduzzi P. Robot-assisted therapy for longterm upper-limb impairment after stroke. N Engl J Med. 2010;362(19):1772-83. [PMID:20400552] http://dx.doi.org/10.1056/NEJMoa0911341

11. Hass CJ, Feigenbaum MS, Franklin BA. Prescription of resistance training for healthy populations. Sports Med. 2001;31(14):953-64. [PMID:11735680] http://dx.doi.org/10.2165/00007256-200131140-00001

12. Kraemer WJ, Adams K, Cafarelli E, Dudley GA, Dooly C, Feigenbaum MS, Fleck SJ, Franklin B, Fry AC, Hoffman JR, Newton RU, Potteiger J, Stone MH, Ratamess NA, Triplett-McBride T; American College of Sports Medicine. American College of Sports Medicine position stand. Progression models in resistance training for healthy adults. Med Sci Sports Exerc. 2002;34(2):364-80.

[PMID:11828249]

http://dx.doi.org/10.1097/00005768-200202000-00027

13. Williams MA, Haskell WL, Ades PA, Amsterdam EA, Bittner V, Franklin BA, Gulanick M, Laing ST, Stewart KJ; American Heart Association Council on Clinical Cardiology; American Heart Association Council on Nutrition, Physical Activity, and Metabolism. Resistance exercise in individuals with and without cardiovascular disease: 2007 update: a scientific statement from the American Heart Association Council on Clinical Cardiology and Council on Nutrition, Physical Activity, and Metabolism. Circulation. 2007;116(5):572-84. [PMID:17638929] http://dx.doi.org/10.1161/CIRCULATIONAHA.107.185214

14. Taylor NF, Dodd KJ, Damiano DL. Progressive resistance exercise in physical therapy: A summary of systematic reviews. Phys Ther. 2005;85(11):1208-23.

[PMID:16253049] 
15. Peterson MD, Rhea MR, Sen A, Gordon PM. Resistance exercise for muscular strength in older adults: A meta-analysis. Ageing Res Rev. 2010;9(3):226-37.

[PMID:20385254]

http://dx.doi.org/10.1016/j.arr.2010.03.004

16. Center of Disease Control. QuickStats: Percentage of adults aged $\geq 18$ years who engaged in leisure-time strengthening activities at least twice a week, by race/ethnicity and sex-National Health Interview Survey, United States, 2009. MMWR Morb Mortal Wkly Rep. 2011; 60(41):1429.

17. Center of Disease Control. Trends in strength trainingUnited States, 1998-2004. MMWR Morb Mortal Wkly Rep. 2006;55(28):770-92.

18. Wu TM, Wang SY, Chen DZ. Design of an exoskeleton for strengthening the upper limb muscle for overextension injury prevention. Mechanism Mach Theory. 2011;46: 1825-39.

http://dx.doi.org/10.1016/j.mechmachtheory.2011.08.003

19. Wu TM, Chen DZ. Dynamic analysis and preliminary evaluation of a spring-loaded upper limb exoskeleton for resistance training with overload prevention. J Mech. 2013; 29(1):35-44. http://dx.doi.org/10.1017/jmech.2012.141

20. The Stock Precision Engineered Components (SPEC) [Internet]. Briston (CT): Associated Spring. Available from: http://springming.so-buy.com/ezfiles/springming/img/img/ 61161/SPEC-04E.pdf

21. Lloyd DG, Besier TF. An EMG-driven musculoskeletal model to estimate muscle forces and knee joint moments in vivo. J Biomech. 2003;36(6):765-76. [PMID:12742444]

http://dx.doi.org/10.1016/S0021-9290(03)00010-1

22. Disselhorst-Klug C, Schmitz-Rode T, Rau G. Surface electromyography and muscle force: Limits in sEMG-force relationship and new approaches for applications. Clin Biomech (Bristol, Avon). 2009;24(3):225-35. [PMID:18849097] http://dx.doi.org/10.1016/j.clinbiomech.2008.08.003

23. Dumas R, Aissaoui R, de Guise JA. A 3D generic inverse dynamic method using wrench notation and quaternion algebra. Comput Methods Biomech Biomed Engin. 2004; 7(3):159-66. [PMID:15512759] http://dx.doi.org/10.1080/10255840410001727805

Submitted for publication December 21, 2012. Accepted in revised form August 7, 2013.

This article and any supplementary material should be cited as follows:

Wu TM, Chen DZ. Biomechanical study of upper-limb exoskeleton for resistance training with three-dimensional motion analysis system. J Rehabil Res Dev. 2014;51(1): 111-26.

http://dx.doi.org/10.1682/JRRD.2012.12.0227

ResearcherID/ORCID: Dar-Zen Chen, PhD: D-1463-2012

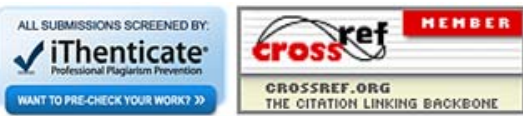

